\title{
Estrategia Sectorial de Innovación para el Encadenamiento Productivo
}

\author{
Strategy of Innovation for the Productive Linkage
}

\author{
José Manuel Solana-Garzón* \\ Fundación Universitaria Tecnológico Comfenalco - \\ Colombia \\ ORCID iD: https://orcid.org/0000-0001-6423-8964 \\ jsolana@tecnologicocomfenalco.edu.co
}

\section{Pablo Herrera-Capdevilla}

Fundación Universitaria Tecnológico Comfenalco Colombia

ORCID iD: https://orcid.org/0000-0003-3951-0676

herrerap@tecnologicocomfenalco.edu.co

* Autor a quien debe ser dirigida la correspondencia
Fecha de recepción: 02/05/2020

Fecha de evaluación: 11/06/2020

Fecha de aceptación: 27/07/2020

\author{
Cómo citar: Solana-Garzón, J., \& Herrera-Capdevi- \\ lla, P. (2020). Estrategia Sectorial de Innovación para \\ el Encadenamiento Productivo. Revista Cientifica Anfi- \\ bios, 3(2), 29-36. https://doi.org/10.37979/afb.2020v3n2.70
}

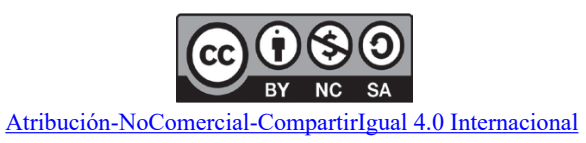

\section{Resumen}

En la actualidad, fenómenos como la globalización y la interdependencia económica de los países ha hecho que los requerimientos en bienes y servicios se encuentren en óptimas condiciones para satisfacer la demanda mundial. Esos requerimientos, han hecho que la economía se oriente hacia los comodities que marcan la pauta mundial como es el caso del Petróleo y sus derivados. Es así como muchas compañías vinculadas a este sector requieren de una estrategia sectorial de innovación acorde con las necesidades actuales. Una de estas estrategias está relacionada con el mantenimiento como eje fundamental para garantizar la operación, es por esto que surgen necesidades fundamentales en las organizaciones que realizan procesos petroquímicos, de contar con proveedores que puedan satisfacer esas necesidades. Es por esto que se propone el diseño de una estrategia sectorial en innovación en el clúster de mantenimiento competitivo con el fin de fortalecer la productividad y competitividad de las organizaciones y actores que hacen parte de este. Los hallazgos más relevantes se vinculan análisis de brechas, evaluación de tendencias tecnológicas y una definición de plan de gestión de tecnologías y de desarrollo del clúster. Esta propuesta se fundamenta en los modelos de encadenamiento productivo y se basa en las teorías de estrategias de investigación e innovación para la especialización inteligente RIS3, capacidades de innovación empresarial, estrategia empresarial.

Palabras clave:

Estrategia; capacidad de innovación; clúster; mantenimiento; encadenamiento productivo.

\begin{abstract}
Currently, phenomena such as globalization and the economic interdependence of countries have made the requirements in goods and services in optimal conditions to meet world demand. These requirements have made the economy to be oriented towards the commodities that set the world standard, as is the case of oil and its derivatives. Thus, many companies linked to this sector require a sectorial strategy of innovation in accordance with current needs. One of these strategies is related to maintenance as a fundamental axis
\end{abstract}


to guarantee the operation, that is why fundamental needs arise in the organizations that carry out petrochemical processes, to have suppliers that can satisfy those needs. That is why we propose the design of a sectorial strategy in innovation in the competitive maintenance cluster in order to strengthen the productivity and competitiveness of the organizations and actors that are part of it. The most relevant findings are linked to gap analysis, evaluation of technological trends and a definition of a technology management plan and cluster development. This proposal is based on the models of productive chaining and is based on the theories of research and innovation strategies for intelligent specialization RIS3, business innovation capabilities, business strategy.

Keywords:

Strategy; innovation capacity; cluster; maintenance; productive linkage.

\section{Introducción}

En la actualidad, muchos países alrededor mundo no poseen una gran producción de petróleo, sin embargo, en muchos casos su balanza comercial internacional, tiene una dependencia muy importante en esta (Solórzano, 2015). De acuerdo a lo anterior, desde hace unos años se ha volcado las tendencias industriales hacia la consecución de nuevos yacimientos de petróleo, pero además a tener la capacidad que transformar esa materia prima en productos intermedios o en productos terminados (Rojas, 2019). Esta dinámica ha generado que en los países con yacimientos de petróleo, se gesten proyectos que puedan dar respuesta a las necesidades del país desde lo industrial, logístico, portuario y astillero (Nguyen, 2020). Estas necesidades han hecho que se realicen grandes inversiones en la construcción y ampliación de puertos marítimos, ampliación de la conectividad terrestre, instalación y ampliación de astilleros entre otras obras para esos propósitos (Hein \& Schubert, 2020)

Con los retos que el contexto anterior supone, se han identificado nuevas necesidades y oportunidades asociadas a negocios y organizaciones relacionadas con el suministro de personal, suministro de materiales para obras civiles, suministro de equipos, suministro de piezas metalmecánicas (Balza-Franco et al., 2017), pero uno de los más importantes en el requerimiento de proveedores, personal y tecnologías enfocadas al mantenimiento de esa infraestructura para garantizar su operación con el cumplimiento de los estándares mínimos mundiales y locales de seguridad física, personal y ambiental que contribuyan al desarrollo sostenible de la ciudades y países (Rentizelas et al., 2018).

Al definir el mantenimiento como eje fundamental para garantizar la operación, surge una necesidad fundamental de las empresas que realizan procesos petroquímicos, de contar con empresas proveedoras que puedan satisfacer esas necesidades de mantenimiento, pero a su vez, que se conviertan en aliados estratégicos para aprovechar las ventajas que se puedan obtener de esto. (Wang et al., 2020)

De acuerdo con Mohseni et al., (2019) los aliados estratégicos, deben tener una orientación en la que estén en la capacidad de prestar los servicios especializados que necesita la organización, así como la tecnología, recursos financieros, requerimientos mínimos, habilidad de ejecutar las actividades y bajos costos o competitivos.

Además de estas necesidades el clúster del Mantenimiento Competitivo también demanda de ciertos requerimientos para un óptimo funcionamiento, por su gran tamaño, requieren de servicios de mantenimiento para sus procesos operacionales (Ospina-Mateus et al., 2017), las organizaciones proveedoras de este clúster, por su parte, son aquellas que por su vocación y estructura, son potenciales ejecutantes o están ejecutando actividades de mantenimiento; y las Entidades de Apoyo son aquellas organizaciones que pueden contribuir o respaldar a la estructuración o desarrollo de esas actividades de mantenimiento(Salas Navarro et al., 2018).

Para que esta iniciativa pueda permanecer en el tiempo, se deben plantear una serie de condiciones y parámetros que puedan ser la base de constitución y ejecución durante su vigencia, es decir, fijar los ejes sobre los cuales se desarrollaran las actividades de conformación y desarrollo, para así cumplir con las características anteriormente descritas. Por consiguiente, se plantea un modelo de proyecto con la participación de todos los actores, para construir la estrategia sectorial del Clúster de Mantenimiento Competitivo que debe entregar las pautas que con- 
tribuyan a cumplir las necesidades de servicios de Mantenimiento de las Empresas Anclas o en dicho caso, de todas aquellas que así lo requieran.

Para estos fines se propone inicialmente una aproximación teórica en la que se esbocen conceptos vinculados con estrategias de innovación y encadenamiento productivo, con énfasis en los clústeres sectoriales. Posteriormente se expone la metodología tomando en cuenta algunos elementos de la prospectiva de tal manera que se pueda proponer un modelo general para organizaciones inmersas en el sector petrolero y sus derivados. Por último, se plantean las conclusiones y discusiones.

\section{Aproximación Teórica}

El marco teórico de referencia para el desarrollo del diseño de la estrategia sectorial del clúster mantenimiento competitivo está basado en las teorías de estrategia de investigación e innovación para la especialización inteligente RIS3, la cual es un modelo para la construcción de estrategias sectoriales de innovación teniendo en cuenta factores internos y externos de las organizaciones para competir. Al igual se tuvieron en cuenta otros modelos sectoriales y territoriales de innovación que han planteado distintos autores en las últimas épocas como los distritos industriales, los clústers industriales, entre otros siendo la estrategia RIS3 la más adecuada para este proyecto.

La estrategia de investigación e innovación para la especialización inteligente RIS3 para Landabaso (2012), parte de la teoría de que la "La innovación no puede ser dictada pero puede ser cultivada" y plantea que una agenda de transformación económica debe basarse en 4 componentes, entre ellas el número limitado de prioridades sobre la base de las propias fortalezas y especialización internacional - evitar la duplicación y la fragmentación en Espacio Europeo de I+D, segundo la ventaja competitiva, movilizar el talento, haciendo coincidir RDT + I con las capacidades, tercero consolidar la estrategia de clústeres y conectividad, desarrollando grupos de clase mundial y proporcionar a desarrollar espacios de variedades relacionadas y por último el liderazgo de colaboración, contar con sistemas de innovación eficaces como un esfuerzo colectivo basado en la colaboración público-privada (cuádruple hélice).
Al igual que la estrategia RIS3 está el modelo de distrito industrial que según Becattini (1979) quien fue uno de los primeros autores que publico sobre distrito tecnológico y que analizó los planteamientos teóricos de Marshall, aporta que este "es el resultado de la conjunción de determinados rasgos socioculturales de una comunidad, de características históricas naturales de un área geográfica y de características técnicas del proceso productivo y el resultado de un proceso de integración dinámica (un círculo virtuoso) entre la división del trabajo en el distrito y el ensanchamiento del mercado de sus productos".

Y para el caso de Porter (1990) plantea otra teoría que es la de los clústers industriales donde resalta que son "grupos de empresas interconectadas, proveedores, industrias relacionadas y de las instituciones especializadas en campos, flujos fijos de bienes y servicios, sino como dinámica, arreglos basados en la creación de conocimiento, los rendimientos crecientes y la innovación en un sentido amplio". No obstante para plantear una estrategia sectorial existen unos factores determinantes de innovación que según Malerba (2002), "los Sistemas sectoriales tienen una base de conocimientos, tecnologías, insumos y la demanda. Los agentes que lo componen son personas y organizaciones en los distintos niveles de agregación, con los procesos de aprendizaje específicos, competencias, estructura organizativa, las creencias, objetivos y comportamientos. Ellos interactúan a través de los procesos de comunicación, el intercambio, la cooperación, la competencia y mando, y sus interacciones son moldeadas por las instituciones. Un sistema sectorial se somete a los procesos de cambio y transformación a través de la co -evolución de sus diversos elementos".

Según distintos autores existen cuatro elementos que son factores determinantes de la innovación, el primer elemento es la capacidad de innovación que es entendida como el conjunto de características, habilidades y conocimientos que permiten desarrollar la estrategia de innovación (Yam et al., 2004), logrando con ello impulsar la dinámica organizacional, sectorial y nacional, mediante componentes complementarios entre sí, como la tecnología, el conocimiento y competitividad entre otras (Licona \& Blanco, 2016) . El segundo es la cultura y el clima que para los autores Rao \& Weinctraub (2010) realizan un 
planteamiento sobre la cultura innovadora, la cual según estos autores se basa en seis bloques básicos: recursos, procesos, valores, conductas, clima y resultados. Estos seis bloques están estrechamente conectados. Por ejemplo, los valores de la empresa tienen un impacto en el comportamiento de las personas, en el clima del lugar de trabajo y define cómo se miden los resultados.

Estos mismos autores afirman en su publicación que cuando se trata de fomentar la innovación, las empresas generalmente han dado mayor importancia a los recursos, procesos e indicadores de resultados - los bloques más orientados a las herramientas y más fáciles de cuantificar.

Sin embargo, han prestado mucha menos atención a los bloques esenciales de la cultura innovadora orientados a las personas y más difíciles de medir-valores, conductas y clima. No sorprende que muchas compañías hayan hecho un mejor trabajo administrando recursos, procesos y resultados de la innovación que en aquellos bloques de la cultura de innovación más orientados a las personas. Muchos ejecutivos han descubierto que los aspectos que involucran los valores y comportamientos de las personas así como el clima del lugar de trabajo son más intangibles y difíciles de manejar. Como dijo un CEO, "los aspectos blandos son los más duros". Estos difíciles "temas de personas" tienen un gran poder en la construcción de la cultura de innovación y la creación de una ventaja competitiva sostenible. El tercer elemento es la vigilancia del entorno que para Vargas \& Castellanos (2005), la esencia de la vigilancia radica en convertirse en un sistema que permita observar y analizar el entorno, de manera que se genere información útil para que las empresas e industrias de los diferentes sectores productivos puedan tomar decisiones y emprender acciones encaminadas hacia la consecución de sus objetivos.

El último elemento es la estrategia de innovación donde; "la innovación no es un fin en sí mismo, sino un medio para alcanzar unos objetivos estratégicos. Como tal, debe situarse en el centro y no en la periferia del desarrollo corporativo, y debe seguir las directrices estratégicas de la compañía. La estrategia pretende definir prioridades tanto en la actuación diaria como en los impulsos de cambio. Son varias las salidas de la reflexión estratégica, pero en el caso concreto de la innovación es importante crear el marco de ejes estratégicos. El primer paso para implantar un sistema de generación de iniciativas estratégicas descansa sobre tres pilares: la creación de un comité de innovación con unas normas de funcionamiento definidas, la implantación de un proceso ordenado de las etapas y la formación de equipos de proyectos de innovación”. (Vilà \& Muñoz, 2004).

\section{Metodología}

Para la construcción de una estrategia sectorial de innovación para el encadenamiento productivo, inicialmente se parte una caracterización de las capacidades de las empresas proveedoras, analizando la oferta y demanda del sector. Seguidamente y con base a esto se determinaron las brechas de las necesidades actuales que demandan los clientes frente a las capacidades actuales de los proveedores. Luego se evaluaron las tendencias tecnológicas y se priorizaron en unas líneas de acción y proyectos, que por último permitieron definir unas acciones estratégicas para el sector.

Para la construcción de la estrategia sectorial, se planteó la metodología de Mesas de Trabajo dinámicas en las que se desarrollan técnicas ágiles para recopilación de información y a partir de ellas se puedan definir las brechas que puedan ser el punto de partida para definir las líneas de acción y los proyectos estratégicos que se deban visionar para así materializar los escenarios futuros que contribuyan al resultado de las metas de cada uno de los actores participantes. La estrategia sectorial, será la carta de navegación que requieren las empresas para poder plantear los cambios necesarios al interior de sus organizaciones que puedan enfocarlas hacia la satisfacción de las necesidades y entrar a participar como empresas de talla mundial. Este proyecto es pertinente para el desarrollo de los sectores apuesta del departamento, porque da soporte a los elementos de apoyo de las cadenas de valor de estos, permite la reducción de costos de las empresas anclas y propende por el aumento de la competitividad del departamento.

Por cada mesa de trabajo realizada se contemplaron ciertas actividades que permitieron la recolección de la información, específicamente para caracterizar a las empresas se construyó un instrumento para las empresas proveedoras y otro para las empresas anclas. Para el caso de las prov- 
eedoras instrumento fue pensado en la estructura y las características de una empresa sostenible en el mundo globalizado y enfocado en servicios de mantenimiento industrial, inicialmente el instrumento tuvo un componente de caracterización, en el cual se organizaron las empresas por subsector dependiendo su actividad económica, tamaño de la empresa, clientes y capacidad productiva.

Para que una empresa sea competitiva, según la teoría de capacidades dinámicas, una empresa debe tener definidos seis componentes, que se enmarcan como una estrategia organizacional definida los cuales son: procesos claves organizados, personal comprometidos y con buena gestión, una estrategia comercial fundamentada en un buen modelo de negocio y capacidades como la gestión de los servicios y productos, y el rendimiento tecnológico de la empresa. Así mismo para el caso de las anclas el instrumento estuvo compuesto de seis elementos donde se tienen una línea base de la gestión de activos, la información se recolecto a través de una entrevista donde se analizaron los siguientes componentes: información básica, dirección de la gestión del mantenimiento, planificación y programación del mantenimiento, control del mantenimiento, necesidades críticas del servicio de mantenimiento y por último la gestión de proveedores Barreto (2010)

\section{Resultados}

\section{Visión prospectiva del 2035 clúster de man- tenimiento}

El horizonte de tiempo definido para el escenario futuro construido fue el año 2035, este fue considerado un espacio propicio para crecer como sector y lograr un desarrollo a nivel nacional e internacional para cualquier organización. Para la construcción de este direccionamiento estratégico se tuvieron en cuenta estudios, trabajos, reuniones con los actores principales del clúster, análisis de información externa a partir de procesos de vigilancia y experiencias de años anteriores.

El proceso realizado de identificación y priorización de las variables estratégicas, fue resultado de un proceso colaborativo y dinámico, en el cual los empresarios primero identificaron factores de cambio en el sector y luego generaron iniciativas transformadoras para el sector.

En este orden de ideas, de este proceso inicialmente resultaron 19 variables y luego de la priorización mediante la matriz de impacto cruzado se determinaron 11 variables reto, las cuales son un factor importante en el aumento de la competitividad del sector, a continuación, se pueden apreciar todas las variables y los vectores del futuro, los cuales fueron identificados por el acercamiento entre las variables.

Tabla 1. Vectores del futuro para el clúster de mantenimiento competitivo

\begin{tabular}{cc}
\hline Variables estratégicas & Direccionadores o vectores de futuro \\
\hline Fortalecimiento empresarial & \\
Formulación de proyectos & \\
Certificaciones & Desarrollo Empresarial \\
Estrategia de marketing & \\
Desarrollo tecnológico empresas anclas & \\
Transferencia de tecnología & \\
Modelo de asociatividad & Integración \\
Comité técnico del sector & \\
Liderazgo empresarial & \\
Modelo de formación por competencias & \\
Gestión del conocimiento & Fuente: Elaboración propia de los autores
\end{tabular}

Fuente: Elaboración propia de los autores

A continuación, se presenta el escenario óptimo para el clúster de mantenimiento competitivo, el cual expresa la importancia de la integración sectorial, como también la inno- vación y el desarrollo tecnológico como factores diferenciadores para poder cumplir con las necesidades de los distintos sectores productivos. 


\section{Escenario Optimo: "Somos Un Clúster Competitivo"}

Corre el año 2035 y el Clúster de Mantenimiento Competitivo se consolida como una estrategia de integración de empresas de talla mundial e instituciones, fundamentadas en la innovación y desarrollo tecnológico, capaces de suplir los bienes y servicios que demandan los sectores productivos de la región y orientados hacia la generación de desarrollo económico y social.

Dentro de los procesos de reflexión realizados, se resaltó la importancia de la permanencia y el compromiso de las empresas anclas, las instituciones como la cámara de comercio, la gobernación la alcaldía en el proceso de la consolidación del clúster de mantenimiento. Los empresarios expresaron que, sin el apoyo y el compromiso de estas instituciones, será muy difícil el éxito del clúster.

De acuerdo con la visión prospectiva plateada a 2035 del desarrollo del clúster de mantenimiento, se construyeron tres hitos claves en este proceso, el primero visionado al 2016 con la consolidación de un sistema de gobernanza que permita dinamizar las estrategias del clúster y una segunda, para los años 2017 y 2018 con un proceso de alistamiento del clúster, apalancado por el proceso de ampliación de la refinería en la ciudad donde se implemente el proyecto y un tercer hito, fundamentado en la visión prospectiva, la cual es consolidar un clúster de mantenimiento competitivo de talla mundial.

\section{Plan estratégico para el alcance de la visión} 2035 del clúster de mantenimiento competitivo.

Para desarrollo de la visión prospectiva, en conjunto con los empresarios se definieron un conjunto de estrategias, las cuales están alineadas al direccionamiento del gobierno, de la empresa financiadora y la cámara de comercio, su construcción al igual que los anteriores, fue resultado de un proceso colaborativo entre la academia, el estado y el sector industrial. A continuación se mencionan las grandes estrategias y el objetivo respectivo de cada uno.

\section{Fortalecimiento empresarial}

Fortalecer las capacidades de las empresas proveedoras de servicios de mantenimiento, proporcionándoles mecanismos y herramientas buscando servicios especializados, competitivos y de alto valor agregado.

\section{Estrategia de marketing}

Diseñar e implementar estrategias de marketing que permitan la visibilidad, la relación entre actores y propicie la interacción entre la oferta y demanda.

\section{Transferencia de tecnología}

Desarrollar procesos de transferencia de Tecnologías que permitan a las empresas proveedoras ofrecer servicios de alta complejidad, mejorar sus procesos productivos y garantizar un estándar de cumplimiento con los requerimientos en materia de tecnologías manejada por los nuevos proyectos de ampliación de las empresas anclas.

\section{Gobernabilidad}

Propiciar un sistema de gobernanza que permita la interacción entre los actores, direccione las estrategias y dinamice una cultura clúster en la industria del mantenimiento competitivo en la ciudad donde se desarrolle el modelo.

Las estrategias definidas anteriormente son acordes a los direccionados estratégicamente definidos para la consolidación del sector, cada uno responde a una necesidad latente para lograr un clúster de mantenimiento de talla mundial. En función a cada eje, también se definieron unas acciones, indicadores y una estrategia de sostenibilidad que permita que este proyecto logre su fin. Cabe resaltar la importancia de la estrategia de gobernabilidad, como la estructura que dinamiza la relación de las instituciones y las empresas vinculadas al proyecto.

\section{Conclusiones}

Este proyecto es resultado de un esfuerzo entre la academia, la industria e instituciones públicas 
de fomento al desarrollo empresarial y su objetivo principal fue el desarrollo de un encadenamiento productivo a través del diseño de una estrategia sectorial de innovación basada en teoría de clúster.

En cuanto al plan estratégico construido, está acorde a la visión prospectiva a 2035 y con los hitos definidos en los próximos años, esta ruta permite crecer competitivamente y coherentemente con su entorno, la ciudad y sus apuestas productivas están crecimiento en gran medida, por lo cual sus empresarios deben crecer proporcionalmente. Al igual, las instituciones de apoyo también deben fortalecer sus estrategias de interfaz, para poder aumentar sus niveles de Investigación y generar nuevo conocimiento para el desarrollo del sector. Los clústeres en la actualidad, son una apuesta de territorio, que permiten generar riquezas a una región, aportando valor y logrando trabajar colaborativamente en función a retos estratégicos. Para la continuación de este proyecto, es necesario la articulación permanente de todas las instituciones y vincular este sector como una estrategia en los planes de desarrollo departamentales, para que puedan verse como apuesta de región.

Una de las discusiones que se generan respecto a los hallazgos propuestos, se basa en el uso de la prospectiva no solamente para las estrategias de innovación de las empresas y clústeres, si no también planes estratégicos que vinculen actores y factores mucho más generales como pueden ser los entes territoriales (Del Rio Cortina et al., 2019), asimismo, también se proponen mejoras en la competitividad de los clústeres por medio de estrategias diferenciadores y generadoras de valor (Del Río Cortina et al., 2018) y finalmente la construcción de la marca de las organizaciones y clusteres por medio de la responsabilidad social empresarial, que al sector petroquímico, debido a su alto impacto ambiental, puede resultar positivo para generar competitividad (Del Río-Cortina et al., 2017)

\section{Referencias}

Balza-Franco, V., Paternina-Arboleda, C. D., Cantillo, V., Macea, L. F., \& Ramírez-Ríos, D. G. (2017). A collaborative supply chain model for non-for-profit networks based on cooperative game theory. International Journal of Logistics Systems and Management, 26(4), 475-496. https://doi.org/https://doi.org/10.1504/IJLSM.2017.082614

Barreto, I. (2010). Dynamic capabilities: A review of the past research and agenda for the future. Journal of management 36 (1): 256-280.

Becattini, G. (1979). «Dal «settore» industriale al «distretto» industriale. Alcune considerazioni sull'unità di indagine dell'economia industriale». Rivista di economia e politica industriale.

Comisión Europea. (2013). Estrategias de investigación e innovación. España.

Del Rio Cortina, J., Arias, C. R., Espitia, N. L., \& Arbeláez, D. C. (2019). Prospective and territorial development in island areas: Empirical evidence from Bocachica-Colombia. Problems and Perspectives in Management, 17(4), 176-189. https://doi.org/10.21511/ppm.17(4).2019.15

Del Río Cortina, J., Cardona Arbelaez, D., \& Pérez Olascuaga, S. J. (2018). Estrategias diferenciadoras y generadoras de valor en economías emergentes. Caso. Saber, Ciencia y Libertad, 13(1), 133-144. https://doi.org/10.18041/2382-3240/saber.2018v13n1.2083

Del Río-Cortina, J. L., Cardona -Arbeláez, D., \& Guacarí-Villalba, A. (2017). Responsabilidad social empresarial y construcción de la marca: una nueva mirada a las estrategias de gestión. Revista De Investigación, Desarrollo E Innovación, 8(1), 49. https://doi.org/10.19053/20278306.v8.n1.2017.7370

Hein, C., \& Schubert, D. (2020). Resilience and Path Dependence: A Comparative Study of the Port Cities of London, Hamburg, and Philadelphia. Journal of Urban History, 1-31. https://doi.org/10.1177/0096144220925098 
Landabaso, M. (2012). "Research and innovation strategies for smart specialisation . Warsaw (Poland): Ministry of Regional Development and European Commi.

Licona, J. M., \& Blanco, L. F. (2016). Emprendimiento e innovación: estado del arte. Estrategia, 2(1), 73-86.

Lundvall, B.-Å. (1997). National Systems and National Styles of Innovation. Fourth International ASEAT Conference.

Malerba, F. (2002). Sectoral systems of innovation and production. Research policy - Elsevier, 247 -264 .

Mohseni, M., Abdollahi, A., \& Siadat, S. H. (2019). Sustainable supply chain management practices in petrochemical industry using interpretive structural modeling. International Journal of Information Systems and Supply Chain Management, 12(1), 22-50.

Nguyen, X. P. (2020). Solutions for navigated safety of super-tankers operating on dinh river traffic-lanes and PTSC port. AIP Conference Proceedings, 2207(February).

Ospina-Mateus, H., Acevedo-Chedid, J., Salas-Navarro, K., Natalie, M.-L., \& Jairo, M.-P. (2017). Model of Optimization of Mining Complex for the Planning of Flow of Quarry Production of Limestone in Multiple Products and with Elements for the Analysis of the Capacity. Sciences in Engineering, 544-555., 742(August 2017), III-IV. https://doi.org/10.1007/978-3-31966963-2 48

Porter, M. (1990). The Competitive Advantage of Nations. Macmillan, London.

Rao, J., \& Weinctraub, J. (2010). ¿Cuán Innovadora es la cultura de su organización? MIT Sloan Management Review, 15 pag.

Rentizelas, A., de Sousa Jabbour, A. B. L., Al Balushi, A. D., \& Tuni, A. (2018). Social sustainability in the oil and gas industry: institutional pressure and the management of sustainable supply chains. Annals of Operations Research, 1-22. https://doi.org/10.1007/s10479-018-2821-3

Rojas, C. D. (2019). “Asentados sobre verdaderos lagos subterráneos de riqueza": Propiedad, fomento y regulación del petróleo en Chile (1914-1945). Historia (Santiago), 52(1), 49-80. https:// doi.org/0.4067/S0717-71942019000100049.

Salas Navarro, K., Chedid, J. A., Caruso, N. M., \& Sana, S. S. (2018). An inventory model of three-layer supply chain of wood and furniture industry in the Caribbean region of Colombia. International Journal of Systems Science: Operations and Logistics, 5(1), 69-86.

Solórzano, C. C. (2015). El petróleo hoy. Economía UNAM, 12(35), 100-105. https://doi.or$\mathrm{g} / 10.1016 / \mathrm{j}$.eunam.2015.09.005

Tidd, J., Bessant, J., \& Pavitt, K. (1997). Managing Innovation; Integrating Technological, Market and Organizational Change. New York: John Wiley \& Sons Ltd.

Wang, C. N., Tsai, H. T., Ho, T. P., Nguyen, V. T., \& Huang, Y. F. (2020). Multi-criteria decision making (MCDM) model for supplier evaluation and selection for oil production projects in vietnam. Processes, 8(2). https://doi.org/10.3390/pr8020134 